\title{
Epitaxial growth of GaSb on V-grooved Si (001) substrates with an ultrathin GaAs stress relaxing layer
}

Qiang Li, Billy Lai, and Kei May Lau

Citation: Appl. Phys. Lett. 111, 172103 (2017);

View online: https://doi.org/10.1063/1.5000100

View Table of Contents: http://aip.scitation.org/toc/apl/111/17

Published by the American Institute of Physics

\section{Articles you may be interested in}

Annealing-induced interfacial atomic intermixing in InAs/GaSb type II superlattices

Applied Physics Letters 111, 172101 (2017); 10.1063/1.4999391

Significantly extended cutoff wavelength of very long-wave infrared detectors based on $\mathrm{InAs} / \mathrm{GaSb} / \mathrm{InSb} / \mathrm{GaSb}$ superlattices

Applied Physics Letters 111, 161101 (2017); 10.1063/1.4998502

Substrate-emitting ring interband cascade lasers

Applied Physics Letters 111, 171101 (2017); 10.1063/1.4989514

GaSb/AlAsSb resonant tunneling diodes with GaAsSb emitter prewells

Applied Physics Letters 111, 171104 (2017); 10.1063/1.4997497

Growth and characterization of high quality N-type GaSb/GaAs heterostructure by IMF growth mode using MOCVD for low power application

Applied Physics Letters 111, 162108 (2017); 10.1063/1.5008737

Influence of quantum confined Stark effect and carrier localization effect on modulation bandwidth for GaNbased LEDs

Applied Physics Letters 111, 171105 (2017); 10.1063/1.4993230

\section{Scilight}

Sharp, quick summaries illuminating the latest physics research

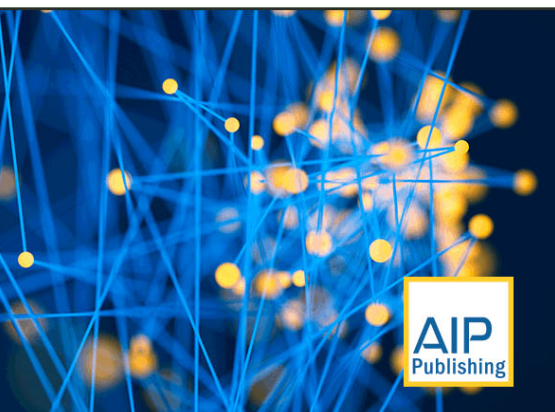




\title{
Epitaxial growth of GaSb on V-grooved Si (001) substrates with an ultrathin GaAs stress relaxing layer
}

\author{
Qiang Li, Billy Lai, and Kei May Lau ${ }^{\mathrm{a}}$ \\ Department of Electronic and Computer Engineering, Hong Kong University of Science and Technology, \\ Clear Water Bay, Kowloon, Hong Kong
}

(Received 14 August 2017; accepted 15 October 2017; published online 26 October 2017)

\begin{abstract}
We report epitaxial growth of GaSb nano-ridge structures and planar thin films on V-groove patterned $\mathrm{Si}$ (001) substrates by leveraging the aspect ratio trapping technique. GaSb was deposited on $\{111\} \mathrm{Si}$ facets of the V-shaped trenches using metal-organic chemical vapor deposition with a $7 \mathrm{~nm}$ GaAs growth initiation layer. Transmission electron microscopy analysis reveals the critical role of the GaAs layer in providing a U-shaped surface for subsequent GaSb epitaxy. A network of misfit dislocations was uncovered at the GaSb/GaAs hetero-interface. We studied the evolution of the lattice relaxation as the growth progresses from closely pitched GaSb ridges to coalesced thin films using x-ray diffraction. The omega rocking curve full-width-at-half-maximum of the resultant $\mathrm{GaSb}$ thin film is among the lowest values reported by molecular beam epitaxy, substantiating the effectiveness of the defect necking mechanism. These results thus present promising opportunities for the heterogeneous integration of devices based on $6.1 \AA$ family compound semiconductors. Published by AIP Publishing. https://doi.org/10.1063/1.5000100
\end{abstract}

GaSb and other $6.1 \AA$ family compound semiconductors are materials of interest for optoelectronics ranging from infrared photodetectors to long wavelength light emitting diodes and interband cascade lasers. However, the current lack of large-size, lattice-matched, and semi-insulating substrates for these devices presents a significant challenge. This, together with emerging opportunities in GaSb nanowire transistors and GaSb-InAs heterojunction tunneling transistors, ${ }^{1,2}$ has stimulated increasing interest in the heteroepitaxy of GaSb. The greatest focus was previously directed towards integrating $\mathrm{GaSb}$ on planar GaAs substrates by leveraging an interfacial misfit (IMF) growth mode. ${ }^{3-6}$ An IMF array, consisting of a 2-dimensional network of misfit dislocations, provides instantaneous strain relief for the $7.8 \%$ lattice mismatch between $\mathrm{GaAs}$ and $\mathrm{GaSb}$, without the need for thick metamorphic buffer layers. Attempts to grow GaSb directly on (001) Si have proved to be challenging due to the even larger lattice mismatch of $12.2 \%$ and the polar-on-nonpolar interface. The prevailing molecular beam epitaxy (MBE) approach is to deposit an ultra-thin AlSb nucleation layer on $\mathrm{Si}^{7-10}$ such that a periodic array of Lomer misfit dislocations, akin to those in the IMF growth of GaSb on GaAs, can be created. However, growing AlSb and related alloys using metal-organic chemical vapor deposition (MOCVD) remains one of the most challenging tasks in all III-V epitaxial material technologies. ${ }^{11}$ To date, very few studies have focused on the direct growth of GaSb on (001) Si by MOCVD. ${ }^{12}$

In this work, we report the growth of GaSb nano-ridges as well as coalesced thin films on exact oriented (001) $\mathrm{Si}$ substrates patterned with nano-V-grooves. The method we employ, selective area growth in conjunction with the aspect ratio trapping (ART) technique, has been utilized in other material systems to effectively reduce crystal defects degrading the quality of hetero-epitaxial films. ${ }^{13-19}$ Here, we first

a)Tel.: (852) 23587049. Fax: (852) 23581485. E-mail: eekmlau@ust.hk introduce a low-temperature-deposited GaAs layer of a few nanometers of thickness to bridge the lattice constant between $\mathrm{Si}$ and GaSb. Highly ordered GaSb nano-ridges free of antiphase-boundaries are demonstrated, thanks to a special stress relaxation mechanism on the $\{111\}$ facets inside the $\mathrm{Si}$ V-grooves. ${ }^{17-19}$ These nano-ridges are coalesced into continuous thin films to yield a template for applications requiring larger device-active regions. The strain relaxation of both the GaSb nano-ridges and the coalesced films is next examined in a range of thicknesses. Finally, the function of oxide stripes in the growth and the crystalline quality are investigated.

Microelectronics standard Si (001) substrates were patterned with $\mathrm{SiO}_{2}$ stripes by dry etching. A brief dip in a $1 \%$ $\mathrm{HF}$ solution was preformed to remove native oxide. Subsequent wet etching in a dilute $\mathrm{KOH}$ solution at $70^{\circ} \mathrm{C}$ created the $\mathrm{V}$-grooves. The samples were then cleaned with a $10 \% \mathrm{HCl}$ solution, rinsed with deionized (DI) water, and immediately loaded into an AIXTRON 200/4 horizontal MOCVD reactor. As illustrated in Fig. 1(a), the dimensions of the oxide stripes were measured to have a linewidth of $55 \mathrm{~nm}$, a spacing of $75 \mathrm{~nm}$, and a height of $145 \mathrm{~nm}$. The growth was carried out using triethylgallium (TEGa), tertiarybutylarsine (TBA), and trimethylantimony (TMSb) as sources. Prior to the III-V epitaxy, the samples were pre-baked in MOCVD at $800{ }^{\circ} \mathrm{C}$ for $15 \mathrm{~min}$ for thermal desorption. The growth began with an ultra-thin GaAs layer nucleated at $400^{\circ} \mathrm{C}$, and then, the temperature was ramped up to the GaSb growth temperature of $525^{\circ} \mathrm{C}$ under an As overpressure. The TBA flux was halted, and the surface was exposed to $\mathrm{H}_{2}$ flux for $30 \mathrm{~s}$ to remove excess As, forming a Ga-rich surface. The first monolayer of GaSb was then grown by pre-flowing TMSb for $60 \mathrm{~s}$ to ensure a distinguishable interface between $\mathrm{GaAs}$ and $\mathrm{GaSb}$. Finally, the TEGa flow was resumed to continue the rest of the GaSb growth. For comparison, direct epitaxy of $\mathrm{GaSb}$ without the ultra-thin GaAs layer exhibited poor uniformity and morphology, as shown in Fig. 1(b). 

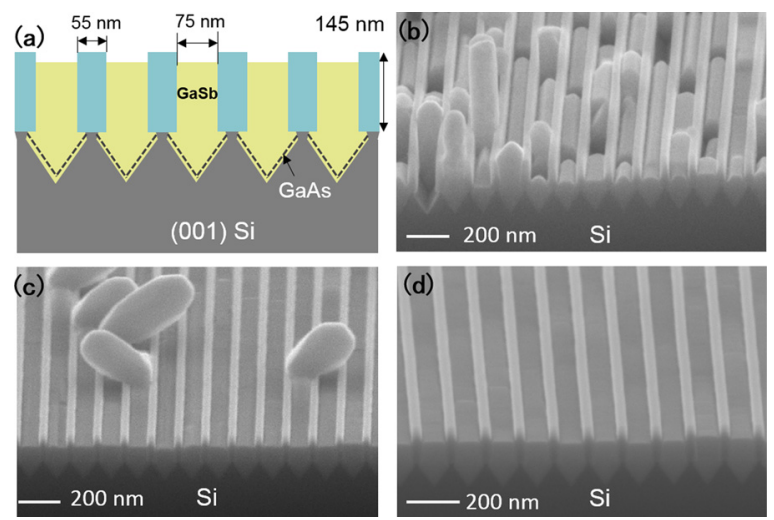

FIG. 1. (a) Schematic and dimensions of GaSb growth on V-grooved $\mathrm{Si}$ using an ultra-thin GaAs stress relaxing layer; (b) Tilted $\left(70^{\circ}\right)$ SEM image of direct GaSb epitaxy on V-grooved Si; (c) and (d) Tilted $\left(70^{\circ}\right.$ ) SEM images of GaSb epitaxy with the GaAs stress relaxing layer at V/III ratios of 1.5 (c) and 1.9 (d).

The GaSb growth temperature, the V/III ratio, and the As desorption time were determined based on optimizations conducted on planar GaAs substrates. V/III ratios of 1.5, 1.7, and 1.9 were attempted, yielding varying results. Figure 1(c) shows a tilted $\left(70^{\circ}\right)$ scanning electron microscopy (SEM) image of the GaSb grown with a V/III ratio of 1.5. The overgrown large crystals originating from the $\mathrm{GaSb}$ nanoridges show faceted sides in addition to clear growth angles. By increasing the $\mathrm{V} / \mathrm{III}$ ratio to 1.9 , these irregular crystals disappear and box-shaped ridges with a flat (001) surface are obtained, as shown in Fig. 1(d). The texture on the surface indicates planar defects, such as stacking faults (SFs), lying in planes perpendicular to the trenches. The ART, unfortunately, cannot prevent this type of defect from reaching the surface.

Figure 2(a) shows a cross-sectional transmission electron microscopy (X-TEM) image of the GaSb ridges with a thickness of approximately $230 \mathrm{~nm}$ measured from the bottom of the $\mathrm{V}$-groove to the GaSb surface. The darker regions in the vicinity of the hetero-interfaces are associated with strain fields in the III-V layer. The strain induced by the lattice mismatch between GaAs and $\mathrm{Si}$ is primarily released through the formation of $\{111\}$ planar defects that terminate at the corner beneath the $\mathrm{SiO}_{2}$ stripes. The upper part of the $\mathrm{GaSb}$ ridges inspected is free of threading dislocations (TDs), despite a few stacking faults. In the zoomed-in view of the V-groove shown in Fig. 2(b), the GaSb and GaAs hetero-interface is clearly distinguishable by the contrast in the image. A Fast Fourier Transform (FFT) image is shown in Fig. 2(c) with the $\mathrm{Si}, \mathrm{GaAs}$, and $\mathrm{GaSb}$ diffraction spots marked along with the associated orientation of the crystal planes. Relative differences in spacing between the three sets of diffraction spots correlate well with their respective lattice constants. From the high resolution X-TEM image in Fig. 2(d), the thickness of the ultrathin GaAs layer on the $\mathrm{Si}$ $\{111\}$ side facet is determined to be around $7 \mathrm{~nm}$. A regularly spaced misfit array is marked by red arrows. Before the onset of GaSb epitaxy, a curved GaAs surface similar to a $\{001\}$ facet was formed at the bottom of the V-groove. The inverse FFT image in Fig. 2(e), which is generated using filtered FFT with $(11 \overline{1})$ and $(\overline{1} \overline{1} 1)$ diffraction spots selected, highlights the array of misfit dislocations presented at the $\mathrm{GaSb} / \mathrm{GaAs}$ interface. On average, the spacing between these misfit dislocations is approximately $14 \mathrm{GaAs}$ lattice sites apart. Likely due to the "U-shaped" GaSb/GaAs interface, the misfit dislocations here are not as highly periodic as the IMF array found on a planar substrate. To fully characterize the crystalline quality, it is reasonable to examine X-TEM along the parallel direction as well. Although there are dislocation-free regions [Fig. 2(f)], threading dislocations are occasionally discovered, as shown in Fig. $2(\mathrm{~g})$. Note that
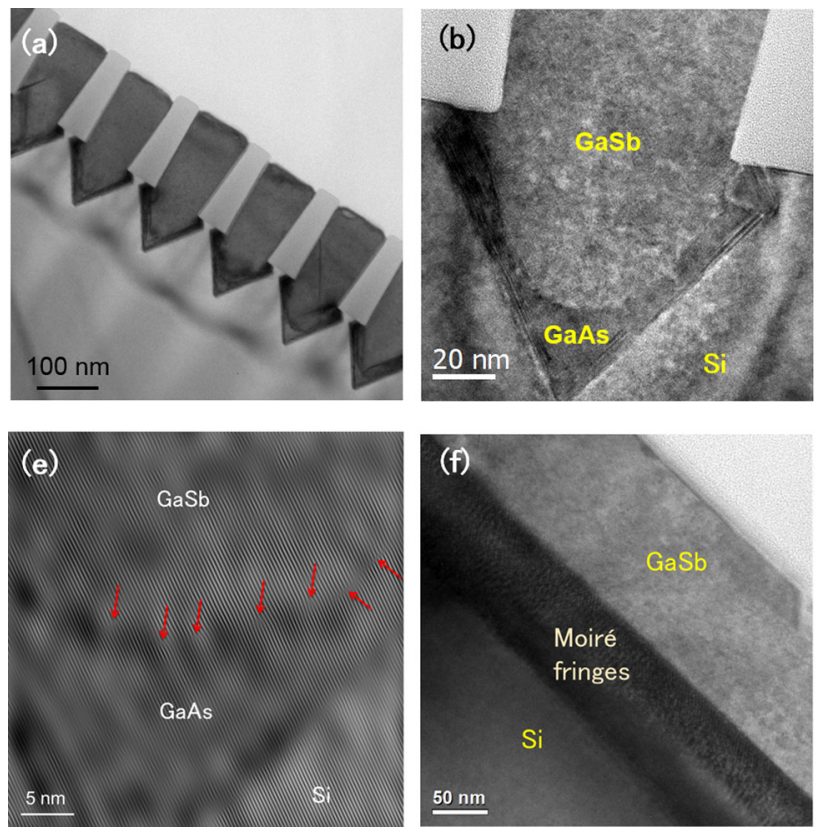
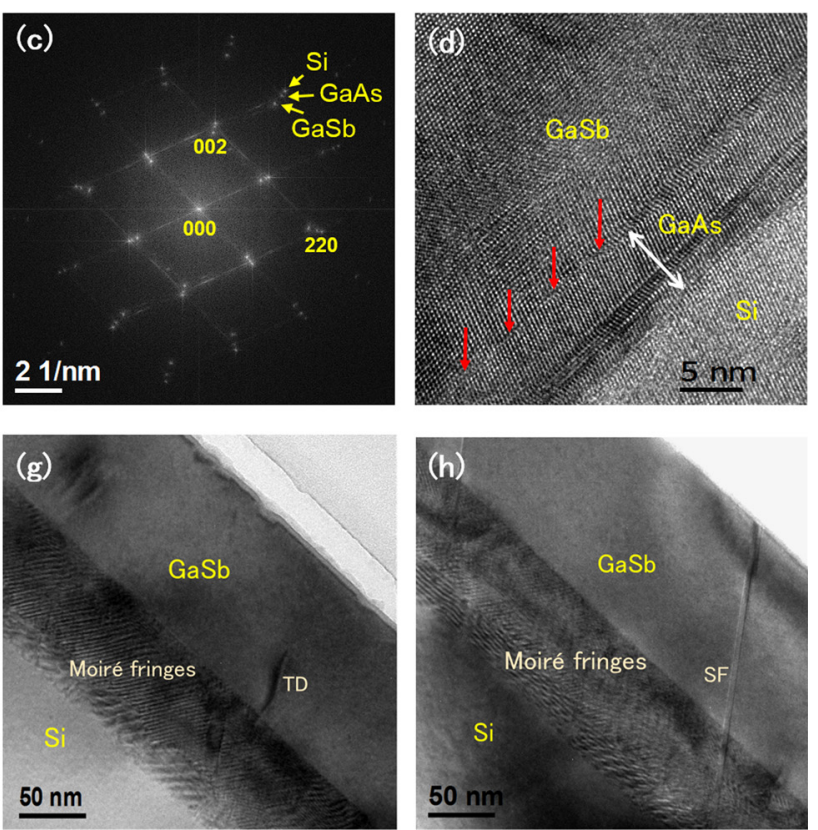

FIG. 2. (a) X-TEM image of GaSb box-shaped ridges on V-grooved Si; (b) X-TEM image of the V-groove showing a "U-shaped" GaSb/GaAs interface; (c) A FFT image showing the diffraction spots for GaSb, GaAs, and Si; (d) A high resolution TEM image showing the GaSb/GaAs/Si hetero-interfaces on the $\{111\}$ Si facet; (e) An inverse FFT image at the bottom of the Si V-groove with an array of misfits marked by red arrows; (f) X-TEM image along the parallel direction showing a dislocation-free region; (g) X-TEM image along the parallel direction showing a TD in GaSb; (h) X-TEM image along the parallel direction showing a stacking fault reaching the GaSb surface. 

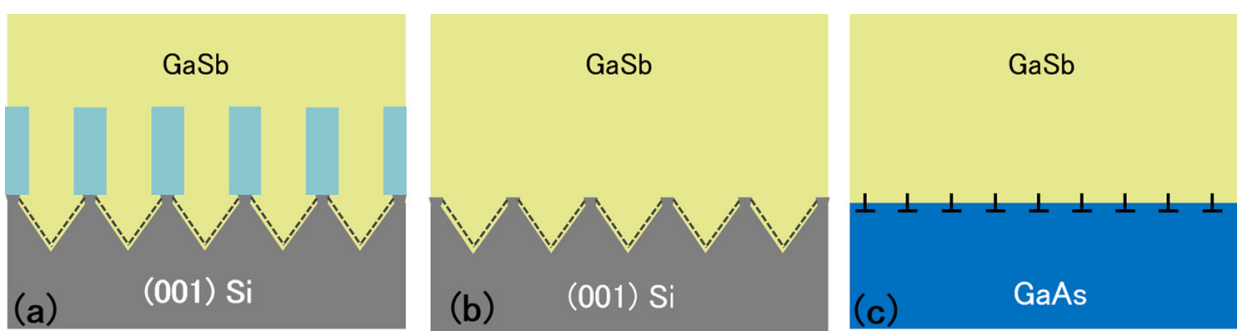

FIG. 3. (a) Schematic of the coalesced $\mathrm{GaSb}$ film with the $\mathrm{SiO}_{2}$ stripes intact; (b) Schematic of the coalesced GaSb film with the $\mathrm{SiO}_{2}$ stripes removed; (c) Schematic of IMF growth of GaSb on $\mathrm{GaAs}$ as a reference sample. these $60^{\circ}$ dislocations may not reach the upper surface because of their propagation towards the oxide sidewall. Only stacking faults in this direction cannot be blocked by the oxide patterns, as seen in Fig. 2(h).

After having investigated the uncoalesced GaSb growth within the trench, coalesced thin films with thicknesses of $150 \mathrm{~nm}$ and $1 \mu \mathrm{m}$ were grown and compared with reference samples of IMF grown GaSb on unpatterned GaAs. Two approaches were designed to merge the nano-ridges. Figure 3(a) shows the schematic previously presented, except that GaSb has now coalesced into a thin film. Taking the uncoalesced GaSb sample, over-etching the $\mathrm{SiO}_{2}$ stripes in buffered oxide etchant, and resuming the growth of $\mathrm{GaSb}$ to form a coalesced layer result in the cross-section shown in Fig. 3(b). Finally, Fig. 3(c) shows the GaSb film grown on GaAs with an IMF array at the hetero-interface.

$\mathrm{X}$-ray diffraction (XRD) measurements were carried out using an Empyrean PANalytical system operating at $40 \mathrm{kV}$ and $40 \mathrm{~mA}$. Figure 4 shows the omega-2theta curves of different thicknesses of $\mathrm{GaSb}$ on $\mathrm{V}$-grooved Si. The noticeable shift in the GaSb peak position as the GaSb film increases in thickness indicates the presence of residual strain. A pronounced shoulder emerges to the left of the main peak of the coalesced $\mathrm{GaSb}$ with embedded $\mathrm{SiO}_{2}$ stripes, aligning with the peak of the uncoalesced GaSb ridges. This is attributed to a superposition of the diffraction from the more compressively strained $\mathrm{GaSb}$ inside the trenches and the coalesced $\mathrm{GaSb}$ above the $\mathrm{SiO}_{2}$ stripes.

Figure 5(a) plots the calculated relaxation percentages based on the relative spacing between the GaSb and Si peaks in the omega-2theta coupled scans. The sample with $\mathrm{SiO}_{2}$ reaches a fully relaxed state at a larger thickness when compared to the sample with $\mathrm{SiO}_{2}$ removed. More interestingly, $\mathrm{GaSb}$ grown on a planar GaAs substrate tends to approach a fully relaxed state in thicker layers, while the samples grown

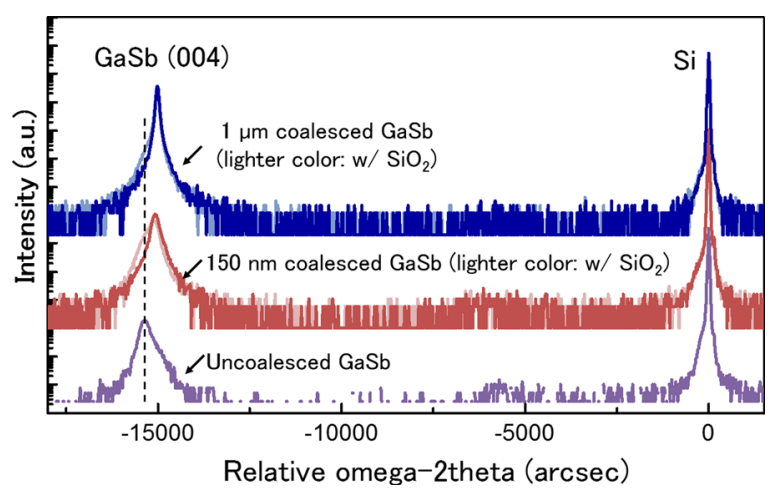

FIG. 4. XRD omega-2theta scans of GaSb grown on V-grooved Si. (The lighter color represents the coalescence with embedded $\mathrm{SiO}_{2}$ stripes.) on $\mathrm{V}$-grooved $\mathrm{Si}$ become tensile strained with a relaxation of over $100 \%$. With linear thermal expansion coefficients of $\mathrm{Si}$, GaAs, and GaSb being $2.6 \times 10^{-6} \mathrm{C}^{-1}, 5.73 \times 10^{-6} \mathrm{C}^{-1}$, and $7.75 \times 10^{-6} \mathrm{C}^{-1}$, respectively, the thermal mismatch between $\mathrm{GaSb}$ and $\mathrm{Si}$ is nearly three times larger compared to that between GaSb and GaAs. The transition from compressive to tensile stress with an increased GaSb thickness on $\mathrm{Si}$ suggests a greater impact of the thermal mismatch. Figure 5(b) compares the omega-2theta full-width-at-halfmaximum (FWHM) of the GaSb peaks. An initial increase in the FWHM of the coalesced GaSb with embedded $\mathrm{SiO}_{2}$ is attributed to the broadening of the Bragg peak by the left shoulder. Growing $1 \mu \mathrm{m}$ coalesced $\mathrm{GaSb}$ on the nano-ridge templates on Si yields a FWHM of 78 and 68 arc sec, corresponding to the samples with and without $\mathrm{SiO}_{2}$, respectively. Figure 5(c) shows the omega rocking curve FWHM values, which are correlated with the total amount of threading dislocations. Benefiting from the epitaxial necking effect from the ART growth process, GaSb on Si only exhibits slightly compromised crystalline quality as compared to the IMF growth of GaSb on GaAs. The FWHM values on both the GaAs and the $\mathrm{Si}$ substrates are among the lowest reported by MBE thus far. $^{10}$

In Fig. 5(c), the observation of similar FWHM values from coalesced $\mathrm{GaSb}$ on $\mathrm{Si}$ regardless of the presence of $\mathrm{SiO}_{2}$ appears to be anomalous, as the effectiveness of the ART concept hinges upon the $\mathrm{SiO}_{2}$ strips for defect trapping. TEM was performed to understand this discrepancy. Figures 6(a) and 6(b) show an X-TEM image of the coalesced GaSb film above the $\mathrm{SiO}_{2}$ stripes. Threading dislocations as well as planar defects originating from the $\mathrm{V}$-grooves terminate at the $\mathrm{SiO}_{2}$ sidewalls. However, the $\mathrm{SiO}_{2}$ stripes also act as nucleation centers for stacking faults and twins, as seen in Fig. 6(b). If these defects do not annihilate within the bulk GaSb, they will eventually reach the sample surface. Looking at the X-TEM image in Fig. 6(c), the removal of the $\mathrm{SiO}_{2}$ stripes prior to the coalescence results in the formation of voids atop the Si ridges. The dashed line in Fig. 6(c) illustrates the contour of the spatially separated $\mathrm{GaSb}$ ridges at the beginning. During the coalescence of GaSb in the areas previously occupied by $\mathrm{SiO}_{2}$, the vertical growth from the bottom undercut regions in conjunction with the lateral growth from the sides of the GaSb ridges prevents overgrowth atop the $\mathrm{Si}$ ridges. Any sources in that region will only contribute to the growth surrounding $\mathrm{Si}$, and once the film has coalesced, the void will be permanently fixed. As seen in Fig. 6(d), the void, being a free surface, allows for the network of defects to terminate, preventing these defects from traveling towards the surface. This peculiar confinement effect of defects thus explains the comparable 

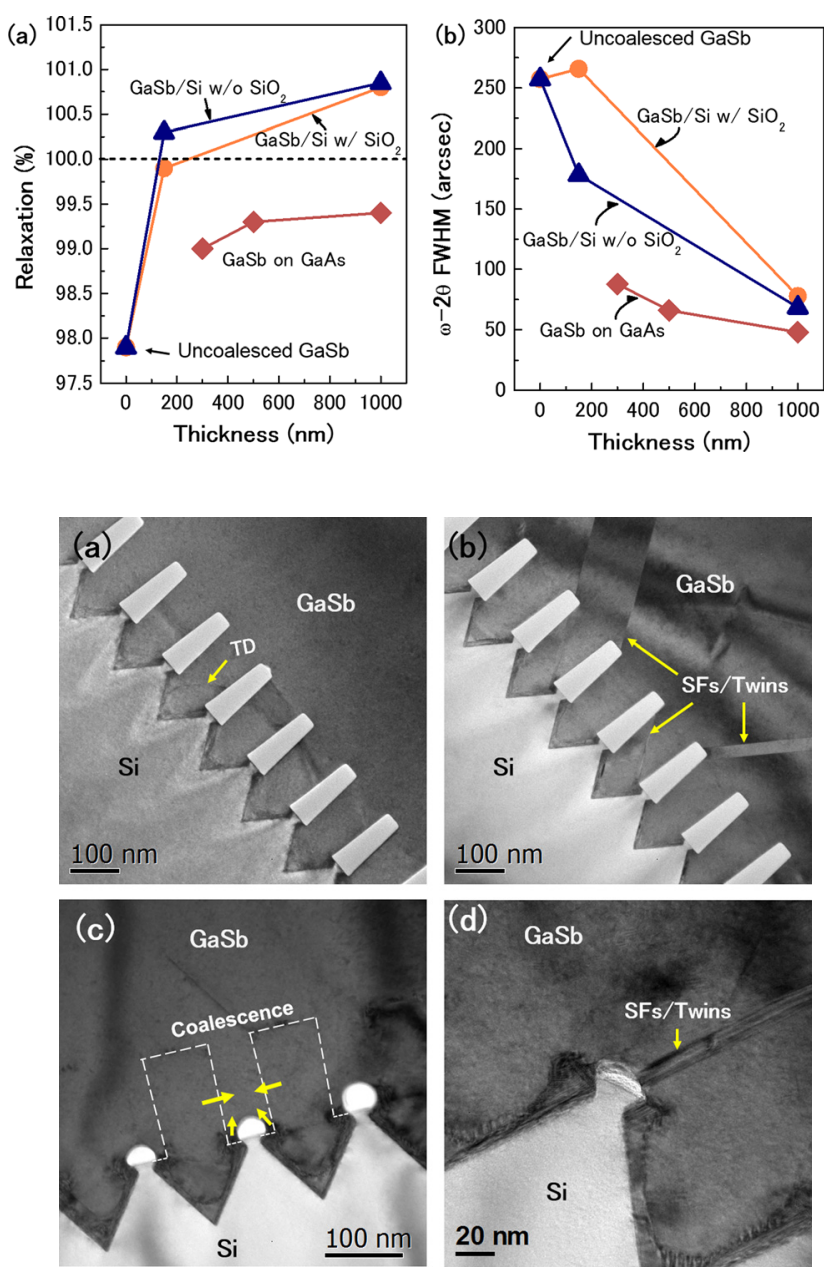

FIG. 6. X-TEM images of the coalesced GaSb film on V-grooved Si with (a)-(b) and without (c)-(d) $\mathrm{SiO}_{2}$ stripes.

threading dislocation density between the samples with and without $\mathrm{SiO}_{2}$ stripes. The ART concept still holds because the entirety of the defects that originated from the $\mathrm{V}$-groove terminated either at the $\mathrm{SiO}_{2}$ sidewall or in the undercut region beneath $\mathrm{SiO}_{2}$. Seemingly ineffectual, removing the $\mathrm{SiO}_{2}$ stripes complicates the growth process and results in no improvements in the XRD FWHM; however, there is a reduction of the stacking faults and twins from the oxides, which is not manifested in the XRD rocking curves. The surface morphology as well as the roughness of the resultant $\mathrm{GaSb}$ thin films on V-grooved $\mathrm{Si}$ is yet to be improved. For a $150 \mathrm{~nm}$ GaSb coalesced thin film, the atomic force microscopy (AFM) measurement shows a root mean square (RMS) roughness of $5.4 \mathrm{~nm}$ across a scan area of $5 \times 5 \mu \mathrm{m}^{2}$.

In conclusion, we have demonstrated the growth of high-quality $\mathrm{GaSb}$ nano-ridges and coalesced films on $\mathrm{V}$ grooved $\mathrm{Si}$ (001) substrates using the aspect ratio trapping method. X-TEM was used to examine the hetero-interface between GaAs and $\mathrm{GaSb}$ in addition to characterizing the propagation of crystal defects in the GaSb films. The influence of different substrates and thicknesses on both the relaxation and the crystalline quality of GaSb was investigated by XRD. With FWHM values comparable with the

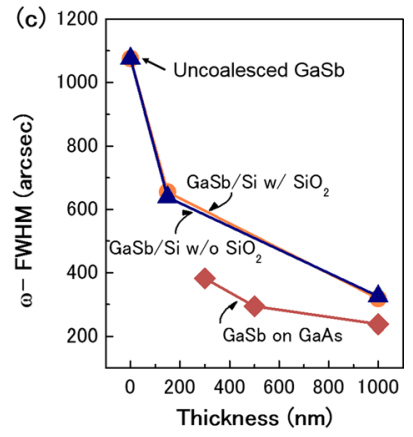

FIG. 5. (a) Lattice relaxation among different substrates and thicknesses; (b) XRD omega-2theta FWHM of the GaSb peak among different substrates and thicknesses; (c) XRD omega FWHM of the GaSb peak among different substrates and thicknesses.

best results of MBE grown GaSb on $\mathrm{Si}$, this study is beneficial in providing an additional method for producing costeffective GaSb templates for future nano-electronic and long wavelength optoelectronic devices.

This work was supported in part by the Innovation Technology Fund of Hong Kong (No. ITS/273/16FP) and an Initiation Grant No. IGN15EG01 from HKUST. The authors would like to thank SUNY Poly for providing the patterned Si substrates and the MCPF of HKUST for technical support. Helpful discussions with Kar Wei Ng, Yu Han, and Bei Shi are also acknowledged.

${ }^{1}$ A. W. Dey, J. Svensson, B. M. Borg, M. Ek, and L.-E. Wernersson, Nano Lett. 12, 5593 (2012).

${ }^{2}$ B. M. Borg, K. A. Dick, B. Ganjipour, M. E. Pistol, L. E. Wernersson, and C. Thelander, Nano Lett. 10, 4080 (2010).

${ }^{3}$ S. H. Huang, G. Balakrishnan, A. Khoshakhlagh, A. Jallipalli, L. R. Dawson, and D. L. Huffaker, Appl. Phys. Lett. 88, 131911 (2006).

${ }^{4}$ J. Tatebayashi, A. Jallipalli, M. N. Kutty, S. H. Huang, G. Balakrishnan,

L. R. Dawson, and D. L. Huffaker, Appl. Phys. Lett. 91, 141102 (2007).

${ }^{5}$ S. Huang, G. Balakrishnan, and D. L. Huffaker, J. Appl. Phys. 105, 103104 (2009).

${ }^{6}$ W. Zhou, W. Tang, and K. M. Lau, Appl. Phys. Lett. 99(22), 221917 (2011).

${ }^{7}$ K. Akahane, N. Yamamoto, S. I. Gozu, and N. Ohtani, J. Cryst. Growth 264, 21 (2004).

${ }^{8}$ Y. H. Kim, J. Y. Lee, Y. G. Noh, M. D. Kim, S. M. Cho, Y. J. Kwon, and J. E. Oh, Appl. Phys. Lett. 88, 241907 (2006).

${ }^{9}$ J. Tatebayashi, A. Jallipalli, M. N. Kutty, S. Huang, K. Nunna, G. Balakrishnan, L. R. Dawson, and D. L. Huffaker, IEEE J. Sel. Top. Quantum Electron. 15, 716 (2009).

${ }^{10}$ J. Rodriguez, K. Madiomanana, L. Cerutti, A. Castellano, and E. Tournié, J. Cryst. Growth 439, 33-39 (2016).

${ }^{11}$ C. A. Wang, J. Cryst. Growth 272, 664 (2004).

${ }^{12}$ T. Orzali, A. Vert, B. O'Brian, J. L. Herman, S. Vivekanand, S. S. Papa Rao, and S. R. Oktyabrsky, J. Appl. Phys. 120, 085308 (2016).

${ }^{13}$ J. Z. Li, J. Bai, J.-S. Park, B. Adekore, K. Fox, M. Carroll, A. Lochtefeld, and Z. Shellenbarger, Appl. Phys. Lett. 91, 021114 (2007).

${ }^{14}$ R. Cipro, T. Baron, M. Martin, J. Moeyaert, S. David, V. Gorbenko, F. Bassani, Y. Bogumilowicz, J. P. Barnes, N. Rochat, and V. Loup, Appl. Phys. Lett. 104, 262103 (2014).

${ }^{15}$ W. Guo, L. Date, V. Pena, X. Bao, C. Merckling, N. Waldron, N. Collaert, M. Caymax, E. Sanchez, E. Vancoille, and K. Barla, Appl. Phys. Lett. 105, 062101 (2014).

${ }^{16}$ C. Merckling, N. Waldron, S. Jiang, W. Guo, N. Collaert, M. Caymax, E. Vancoille, K. Barla, A. Thean, M. Heyns, and W. Vandervorst, J. Appl. Phys. 115, 023710 (2014).

${ }^{17}$ Q. Li, K. W. Ng, and K. M. Lau, Appl. Phys. Lett. 106, 072105 (2015).

${ }^{18}$ Q. Li, Y. Han, X. Lu, and K. M. Lau, IEEE Electron Device Lett. 37, 24 (2016).

${ }^{19}$ Y. Han, Q. Li, S. P. Chang, W. D. Hsu, and K. M. Lau, Appl. Phys. Lett. 108, 242105 (2016). 